\title{
A Hybrid Temporal-Spatio Forecasting Approach for Passenger Flow Status in Chinese High-Speed Railway Transport Hub
}

\author{
Zhengyu Xie, ${ }^{1,2}$ Limin Jia, ${ }^{2}$ Yong Qin, ${ }^{2}$ and Li Wang' \\ ${ }^{1}$ School of Traffic and Transportation, Beijing Jiaotong University, Beijing 100044, China \\ ${ }^{2}$ State Key Laboratory of Rail Traffic Control and Safety, Beijing Jiaotong University, Beijing 100044, China \\ Correspondence should be addressed to Limin Jia; jialm@vip.sina.com
}

Received 16 July 2013; Revised 4 October 2013; Accepted 18 October 2013

Academic Editor: Wuhong Wang

Copyright ( $\odot 2013$ Zhengyu Xie et al. This is an open access article distributed under the Creative Commons Attribution License, which permits unrestricted use, distribution, and reproduction in any medium, provided the original work is properly cited.

With the rapid development of high-speed railway in China, high-speed railway transport hub (HRTH) has become the highdensity distribution center of passenger flow. In order to accurately detect potential safety hazard hidden in passenger flow, it is necessary to forecast the status of passenger flow. In this paper, we proposed a hybrid temporal-spatio forecasting approach to obtain the passenger flow status in HRTH. The approach combined temporal forecasting based on radial basis function neural network (RBF NN) and spatio forecasting based on spatial correlation degree. Computational experiments on actual passenger flow status from a specific bottleneck position and its correlation points in HRTH showed that the proposed approach is effective to forecast the passenger flow status with high precision.

\section{Introduction}

As main influence factors for the safety and sustainability of transportation system, the insecure behaviors and statuses of people are hot issues and difficult problems in traffic safety engineering $[1,2]$. With the rapid development of high-speed railway in China, HRTH has become an interface of multitransportation which includes high-speed railway, civil aviation, highway, waterway, urban rail transit, public transport, and private vehicles, and the safety of passenger flow in HRTH has attracted more and more attention. As the vital node of passenger transport net, HRTH is an important collection and distribution center of various transportation modes and massive passenger flow. The distribution quantity of passengers will be sustained to sharply increase with the growth of high-speed railway operation mileage. As the dramatic increase of passengers, high-density passenger flow is generated, which imposed a rigorous challenge to the safety management of HRTH.

In order to avoid and solve the problems caused by passenger flow abnormal status, many approaches are proposed in literatures which can be mainly classified into two categories. The first category is the studies on passenger flow modeling and simulating in transport hubs. Gipps and Marksjö [3] focused on the prediction the alteration passenger flow in the passing environment and proposed a model for the interactions between passengers which is intended for use in a graphical computer simulation. Seyfried et al. [4] analyzed the influence of various approaches for the interaction between the passengers on the resulting velocitydensity relation based on a modified social force model. Jia et al. [5] analyzed the characteristics of passenger flow and present parameters relation models for passenger flow on different terminal facilities were established based on data statistics. Ji et al. [6] proposed a cell-based model including two steps. The first step is to update speed, which is the cells the passenger can move in one time interval and the other is to analyze the overtaking. Wang et al. [7] simulated the passenger flow in a station hall during the spring festival by modifying the social force model. According to the studies on the first category, decision makers can adjust layout and distribution of facilities and equipment in transport hub to adapt to the modeling and simulating results of passenger 
flow. This category studies aim at decreasing and preventing passenger flow abnormal status before they happened.

The other category focuses on the modeling and simulating of congestion evacuations which are caused by highdensity passenger flow. Zhong et al. [8] introduced the passenger evacuation design in the construction of metros in China and proposed a dynamic model to simulate the passenger evacuation. Jiang et al. [9] studied the effect of varying maximum upstairs speed and the average minimum width of staircase utilized per person by taking two subway stations in China as examples. VanLandegen and Chen [10] integrated a pedestrian model with a Metrorail transit model to evaluate the performance of the Metrorail in the hypothetical large-scale evacuations. Shi et al. [11] firstly investigated and established the safety strategy of evacuation in metro stations, which involves the occupants needed to be evacuated, the evacuation route, and the safety zone. Based on the studies on the second category, decision makers can make an efficient emergency evacuation decision to determine routes and channels of evacuation. This category studies aim at mitigating passenger flow abnormal status after they happened.

According to the literature review above, most studies focus on solving approaches before or after passenger flow abnormal status happened. Specific literature on realtime changing process of passenger flow status is scarce because of limitation from the difficult acquisition of realtime passenger flow status. With the widespread applications of intelligent video surveillance in Chinese HRTH, real-time acquisition of passenger flow status has become feasible. In this paper, passenger flow status is defined as the amount, velocity, and density of passenger flow. We consider the real-time passenger flow status in bottleneck positions of HRTH and propose a hybrid temporal-spatio forecasting approach to reflect the change of passenger flow status.

The rest of paper is organized as follows: a hybrid temporal-spatio forecasting approach for passenger flow status of bottleneck positions is developed in Section 2. Computational results are reported in Section 3 and finally Section 4 covers the conclusion.

\section{A Hybrid Temporal-Spatio Forecasting Approach}

A hybrid forecasting approach for passenger flow status of bottleneck positions is proposed in this section, which combines temporal and spatio forecasting methods. A temporal forecasting based on RBF NN is proposed to forecast passenger flow status of bottleneck position by using the real-time passenger flow status of the position. The temporal forecasting method can rapidly and precisely reflect the passenger flow status in the bottleneck position but is insensitive for the passenger flow fluctuation from correlation points. So we introduce a spatio forecasting approach based on spatial correlation degree to combine with the temporal forecasting approach for improving the forecasting precision.

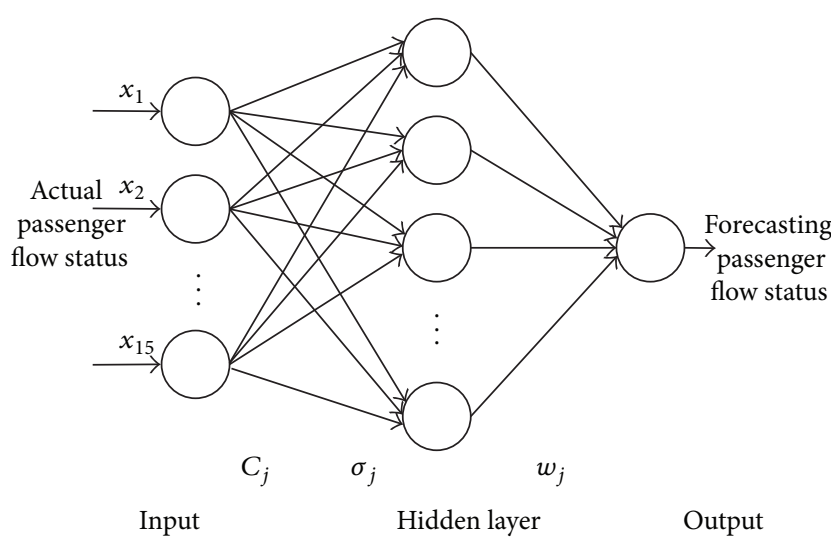

FIGURE 1: Structure of RBF NN.

2.1. Temporal Forecasting Based on RBF NN. The RBF NN is a typical feed-forward neural network, which has many merits, such as nonlinear mapping characteristics, self-organized study ability, training fast, and the capability of converging in global optimization and approaching the function in the best way. Simply for its great advantages, RBF NN has been applied in many fields [12-15]. A temporal forecasting approach based on RBF NN is proposed in this section to forecast passenger flow status of bottleneck positions in HRTH.

2.1.1. Design of RBF NN. The structure of RBF NN is comprised of three different layers: an input layer, a hidden layer and an output layer. The structure of RBF NN for temporal forecasting is shown in Figure 1.

The input vector $X=\left(x_{1}, x_{2}, \ldots, x_{15}\right)$ is composed of the input signals $x_{i}$, and there are fifteen input neurons in our RBF NN. The output $h_{j}(X)$ of the $j$ th neuron in the hidden neuron in the hidden layer is described by

$$
h_{j}(X)=\varphi\left(\left\|X-C_{j}\right\|\right),
$$

where $\varphi(\cdot)$ is the radial basis function, $C_{j}\left(C_{j} \in R^{n}\right)$ is the center of the $j$ th hidden neuron, and $\|\cdot\|$ is the Euclidean norm. In our RBF NN, $\varphi(\cdot)$ is adopted to be the Gaussian function, and $h_{j}(X)$ is described by

$$
h_{j}(X)=\exp \left(-\frac{\left\|X-C_{j}\right\|^{2}}{2 \sigma_{j}^{2}}\right),
$$

where $\sigma_{j}$ is the spread of the $j$ th hidden neuron for the $i$ th input signal.

The output $Y(X)$, which is designed as passenger flow status forecasting, is the linearly combined signal of the outputs $h_{j}(X)$ from the hidden layer with the synaptic weights $w_{j}$ described by

$$
Y(X)=\sum_{j=1}^{m} w_{j} h_{j}(X)
$$

where $m$ is the number of hidden neurons. 
TABLE 1: Mapping structure between input variables and output variable.

\begin{tabular}{lc}
\hline Input variables & Output variable \\
\hline$x_{1}, x_{2}, \ldots, x_{15}$ & $x_{16}$ \\
$x_{2}, x_{3}, \ldots, x_{16}$ & $x_{17}$ \\
$\vdots$ & $\vdots$ \\
$x_{l-15}, x_{l-14}, \ldots, x_{l-1}$ & $x_{l}$ \\
\hline
\end{tabular}

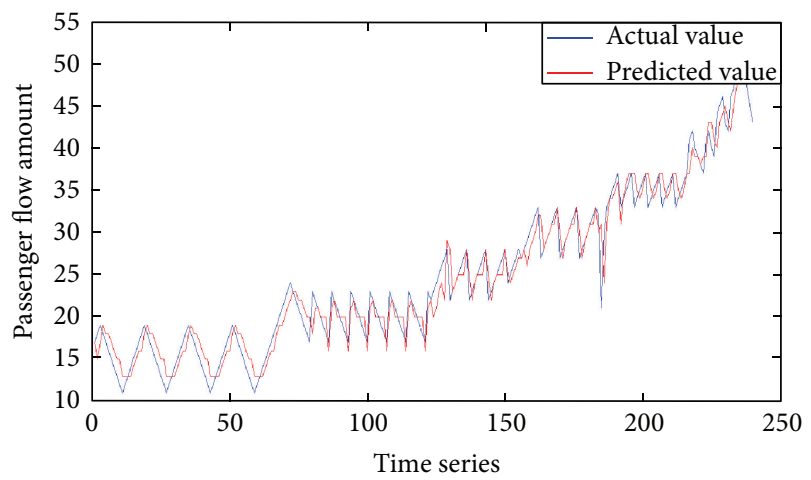

Figure 2: Comparison between actual value and forecasting value based on RBF NN.

2.1.2. Input Variables Determined. It is very important to select proper input variables for a neural network. On the one hand, as the number of the input variables increases, the NN architecture will be larger and the computing time will be longer. On the other hand, the irrelevant or mutually correlated input variables are not useful for improving the prediction accuracy. Therefore, how to select a few but sufficient input variables is a key issue.

According to plenty of experiments on the input variables, we choose passenger flow status (amount, velocity, or density) of 15 points in the time series as the variables input our RBF NN and passenger flow status (amount, velocity, or density) of the point after the 15th point as the output variable. $X=\left\{x_{i} \mid x_{i} \in N^{+}, i=1,2, \ldots, l\right\}$ is a time series set and $x_{i}$ is the passenger flow status (amount, velocity, or density) of the $i$ th point in the time series. The mapping structure between input variables and output variable is shown in Table 1.

2.1.3. RBF NN Testing. In order to test the accuracy of temporal forecasting, we choose 250 actual passenger flow amounts of one bottleneck position in time series as testing samples. The input and output values after normalization are shown in Table 2.

A comparison between actual value and forecasting value based on RBF NN is shown in Figure 2. The average precision of forecasting is $94.14 \%$. The testing result indicates that the $\mathrm{RBF} N \mathrm{NN}$ has a desirable performance on forecasting.

2.2. Spatio Forecasting Based on Spatial Correlation Degree. According to the spatial correlation degree and passenger flow status of bottleneck position $P_{0}$ and its correlation points
TABLE 2: Input and output values.

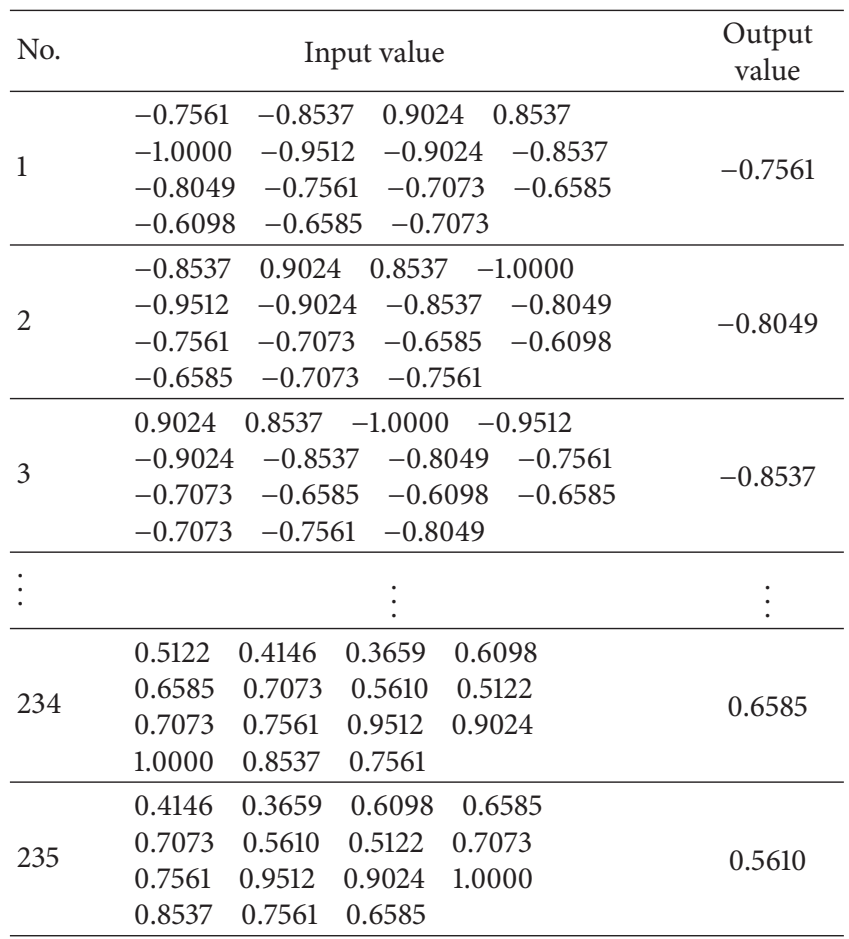

$C_{i}$, a spatio forecasting model is proposed to forecast the passenger flow status of bottleneck position $P_{0}$.

2.2.1. Analysis of Spatial Correlation Degree. $X_{i}=\left\{x_{i}(k) \mid\right.$ $k=1,2, \ldots, n\}(i=1,2, \ldots, m)$ is a sequence set, $x_{0}(k)$ is a reference sequence, and $x_{i}(k)$ is comparison sequence. $r\left(x_{0}(k), x_{i}(k)\right)$ is the correlation coefficient of comparison sequence relative to reference sequence at period $k$, as shown in (4). Consider

$$
\begin{aligned}
r & \left(x_{0}(k), x_{i}(k)\right) \\
& =\frac{\min _{i} \min _{k}\left|x_{0}(k)-x_{i}(k)\right|+\zeta \max _{i} \max _{k}\left|x_{0}(k)-x_{i}(k)\right|}{\left|x_{0}(k)-x_{i}(k)\right|+\zeta \max _{i} \max _{k}\left|x_{0}(k)-x_{i}(k)\right|},
\end{aligned}
$$

where $\zeta$ is the discriminating coefficient, $\zeta \in(0,1)$.

$r\left(x_{0}, x_{i}\right)$ is the correlation degree of the $i$ th comparison sequence $x_{i}$ relative to reference sequence $x_{0}$, as shown in (5). Consider

$$
r\left(x_{0}, x_{i}\right)=\frac{1}{n} \sum_{k=1}^{n} r\left(x_{0}(k), x_{i}(k)\right) .
$$

$\alpha_{i}$ is the correlation weight coefficient and is described by (6). Consider

$$
\alpha_{i}=\frac{r\left(x_{0}, x_{i}\right)}{\sum_{j=1}^{n} r\left(x_{0}, x_{j}\right)} .
$$

2.2.2. Spatio Forecasting Model. According to the passenger flow status of $C_{i}$ and the spatio correlation degree between $P_{0}$ 
and $C_{i}$, the passenger flow status of $P_{0}$ is forecasted based on spatio forecasting model. The initial forecasting value $x_{c}^{\prime}(t)$ of $P_{0}$ at period $t$ is calculated by (7). Consider

$$
x_{c}^{\prime}(t)=\sum_{i=1}^{n} \alpha_{i} x_{i}\left(t-t_{i}\right)
$$

where $x_{i}(t)$ is passenger flow status of $C_{i}$ at period $t, t_{i}$ is the average moving time from $C_{i}$ to $P_{0}$, and $\alpha_{i}$ is the correlation weight coefficient between correlated points $C_{i}$ and congested point $P_{0}$. According to (6), $\alpha_{i}$ is described by

$$
\alpha_{i}=\frac{r\left(P_{0}, C_{i}\right)}{\sum_{j=1}^{n} r\left(P_{0}, C_{i}\right)},
$$

where $r\left(P_{0}, C_{i}\right)$ is the correlation degree of between $C_{i}$ and $P_{0}$.

In order to reduce the errors which are caused by uncertainties passenger flow status, we use the change of initial forecasting value $x_{c}{ }^{\prime}(t)$ and actual passenger flow status value $x_{c}(0)$ of $P_{0}$ at the beginning of period $t$ to describe the forecasting value $x_{c}(t)$ of $P_{0}$ at period $t$. The spatio forecasting model of passenger flow status is shown in (9). Consider

$$
x_{c}(t)=\sum_{i=1}^{n} \alpha_{i} x_{i}\left(t-t_{i}\right)-\sum_{i=1}^{n} \alpha_{i} \mathrm{x}_{i}(0)+x_{c}(0) .
$$

The velocity in correlated points prominently affects the density of congested point; the faster the velocity of correlated points, the weaker affection on the density of congested point. So we adopt velocity to improve the density forecasting. The spatio forecasting model of passenger flow density is shown in (10). Consider

$$
x_{\rho c}(t)=\sum_{i=1}^{n} \frac{k v_{i}\left(t-t_{i}\right)}{v_{0}\left(t-t_{i}\right)} \alpha_{i} x_{\rho i}\left(t-t_{i}\right)-\sum_{i=1}^{n} \alpha_{i} x_{\rho i}(0)+x_{\rho}(0),
$$

where $x_{\rho c}(t)$ is the forecasting passenger flow density of $P_{0}$ at period $t, v_{i}\left(t-t_{i}\right)$ is the velocity of correlated points at period $t-t_{i}, v_{0}\left(t-t_{i}\right)$ is the velocity of congested point at period $t-t_{i}$, and $x_{\rho}(0)$ is the actual density of $P_{0}$ at the beginning of period $t$.

Similarly, the density in correlated points prominently affects the velocity of congested point; the higher the density of correlated points, the faster the velocity of congested point. So, we adopt density to improve the velocity forecasting. The spatio forecasting model of passenger flow velocity is shown in (11). Consider

$$
x_{v \mathcal{c}}(t)=\sum_{i=1}^{n} \frac{k \rho_{0}\left(t-t_{i}\right)}{\rho_{i}\left(t-t_{i}\right)} \alpha_{i} x_{v i}\left(t-t_{i}\right)-\sum_{i=1}^{n} \alpha_{i} x_{v i}(0)+x_{\nu}(0),
$$

where $x_{\nu c}(t)$ is the forecasting passenger flow velocity of $P_{0}$ at period $t, \rho_{i}\left(t-t_{i}\right)$ is the density of correlated points at period $t-t_{i}, \rho_{0}\left(t-t_{i}\right)$ is the density of congested point at period $t-t_{i}$, and $x_{v}(0)$ is the actual velocity of $P_{0}$ at the beginning of period $t$.
2.3. Temporal-Spatio Forecasting Combination. A hybrid forecasting approach to combine the spatio and temporal forecasting is proposed in this section. The forecasting value $x_{r}(t)$ based on RBF NN and the forecasting value $x_{c}(t)$ based on spatioforecasting model are combined by a linear regression model, which is shown in (12). Consider

$$
f(t)=\beta_{1} x_{r}(t)+\beta_{2} x_{c}(t)+\beta_{3} .
$$

The parameters of the model are determined by numerical fitting of actual passenger flow status values and forecasting values generated by spatio and temporal forecasting methods.

According to the spatio and temporal forecasting methods and the combination model, the temporal-spatio forecasting model of passenger flow density is described by

$$
\begin{aligned}
f_{\rho}(t)=\beta_{\rho 1} x_{\rho r}(t)+\beta_{\rho 2}\left[\sum_{i=1}^{n} \frac{k v_{i}\left(t-t_{i}\right)}{v_{0}\left(t-t_{i}\right)} \alpha_{i} x_{\rho i}\left(t-t_{i}\right)\right. \\
\left.\quad-\sum_{i=1}^{n} \alpha_{i} x_{\rho i}(0)+x_{\rho}(0)\right]+\beta_{\rho 3} .
\end{aligned}
$$

According to the spatio and temporal forecasting methods and the combination model, the temporal-spatio forecasting model of passenger flow velocity is described by

$$
\begin{array}{r}
f_{\nu}(t)=\beta_{\nu 1} x_{v r}(t)+\beta_{\nu 2}\left[\sum_{i=1}^{n} \frac{k \rho_{0}\left(t-t_{i}\right)}{\rho_{i}\left(t-t_{i}\right)} \alpha_{i} x_{v i}\left(t-t_{i}\right)\right. \\
\left.\quad-\sum_{i=1}^{n} \alpha_{i} x_{v i}(0)+x_{v}(0)\right]+\beta_{\nu 3} .
\end{array}
$$

\section{Computational Experiments}

To illustrate the proposed forecasting approach, computational experiments are performed by using the actual passenger flow status from a specific bottleneck position in the Chinese HRTH. In order to assess the improvement of our approach, the forecasting of passenger flow density and velocity among temporal forecasting based on RBF NN, spatio forecasting based on spatial correlation degree, and hybrid temporal-spatio forecasting approach are compared. These numerical experiments are performed based on a personal computer with Intel Core(TM) i5-2450M @ $2.50 \mathrm{GHz}$ processors and 4 GB RAM.

To implement the proposed forecasting approach, the parameters related to specific bottleneck position of HRTH are needed. The specific bottleneck position is a ticket entrance $P$ with 3 correlation points $A, B$, and $C$. The average passenger moving time of $A$ to $P$ is $35 \mathrm{~s}, B$ to $P$ is $55 \mathrm{~s}$, and $C$ to $P$ is 20 s. We choose 311 data of passenger flow density and 311 data of passenger flow velocity as the computational examples.

According to RBF NN designed in Section 2, temporal forecasting experiments on passenger flow density and 


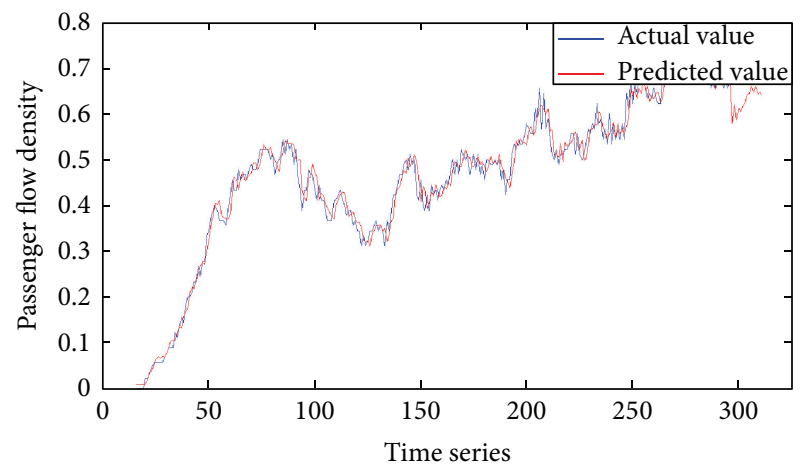

FIGURE 3: Comparison between actual value and forecasting value of passenger flow density based on RBF NN.

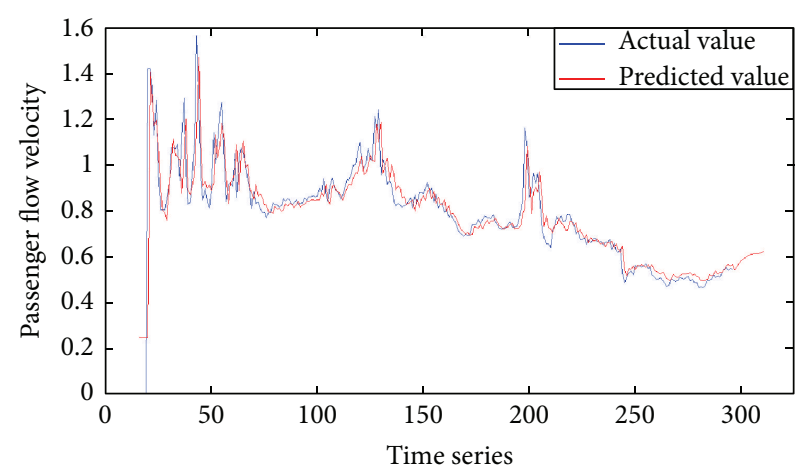

FIGURE 4: Comparion between actual value and forecasting value of passenger flow velocity based on RBF NN.

velocity are implemented. The comparison between actual value and forecasting value of passenger flow density based on RBF NN is shown in Figure 3 and comparison between actual value and forecasting value of passenger flow velocity based on RBF NN is shown in Figure 4.

According to spatio forecasting model proposed in Section 2, spatio forecasting experiments on passenger flow density and velocity are implemented. $x_{0}$ is the passenger flow status sequence of $P, x_{1}$ is the passenger flow status sequence of $A, x_{2}$ is the passenger flow status sequence of $B$, and $x_{3}$ is the passenger flow status sequence of $C$. The spatial correlation degrees are calculated according to (4) and (5). The calculation result is shown as follows:

$$
r(0,1)=0.510, \quad r(0,2)=0.761, \quad r(0,3)=0.404
$$

The forecasting value of passenger flow density based on spatio forecasting model is calculated by (16) and the

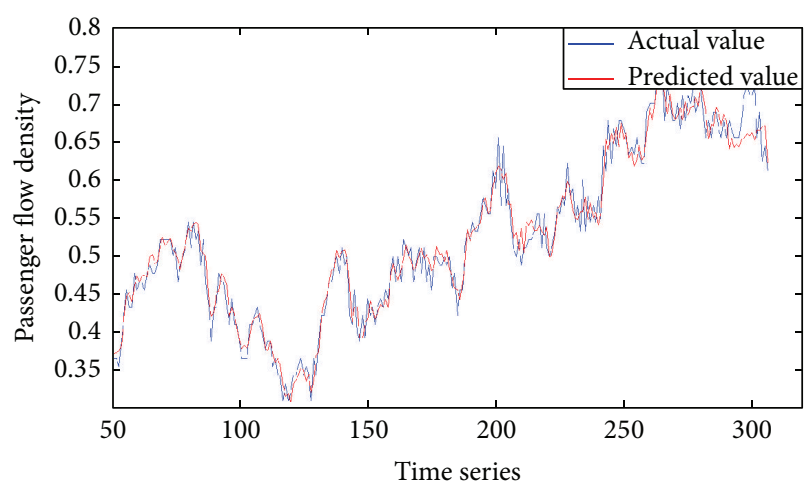

FIgURE 5: Comparion between actual value and forecasting value of passenger flow density based on spatio forecasting model.

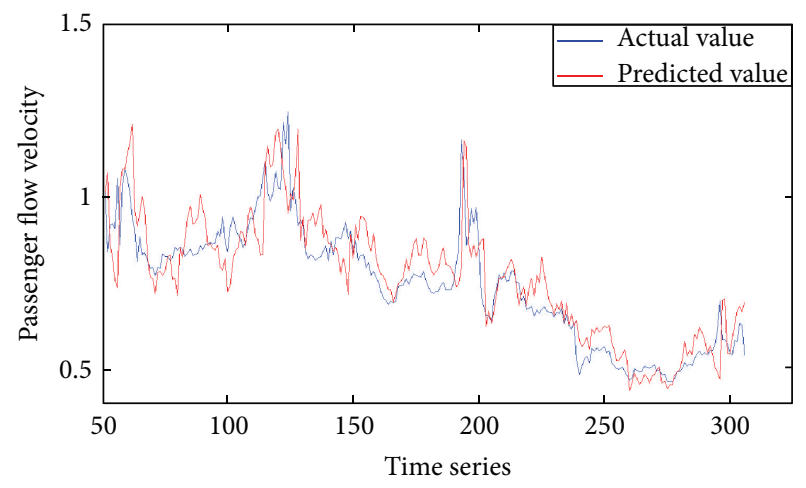

FIGURE 6: Comparion between actual value and forecasting value of passenger flow velocity based on spatio forecasting model.

forecasting value of passenger flow velocity based on spatio forecasting model is calculated by (17). Consider

$$
\begin{aligned}
x_{\rho c}(t)= & \sum_{i=1}^{n} \frac{k v_{i}}{v_{0}} \alpha_{i} x_{\rho i}\left(t-t_{i}\right)-\sum_{i=1}^{n} \alpha_{i} x_{\rho i}(0)+x_{\rho}(0) \\
= & \frac{0.304 v_{1}}{v_{0}} x_{\rho 1}(t-35)+\frac{0.454 v_{2}}{v_{0}} x_{\rho 2}(t-55) \\
& +\frac{0.241 v_{3}}{v_{0}} x_{\rho 3}(t-20)+0.206, \\
x_{\nu c}(t)= & \sum_{i=1}^{n} \frac{k \rho_{0}}{\rho_{i}} \alpha_{i} x_{v i}\left(t-t_{i}\right)-\sum_{i=1}^{n} \alpha_{i} x_{v i}(0)+x_{\nu}(0) \\
= & \frac{0.213 \rho_{1}}{\rho_{0}} x_{\nu 1}(t-35)+\frac{0.363 \rho_{2}}{\rho_{0}} x_{\nu 2}(t-55) \\
& +\frac{0.193 \rho_{3}}{\rho_{0}} x_{\nu 3}(t-20)+0.671 .
\end{aligned}
$$

The comparison between actual value and forecasting value of passenger flow density based on spatio forecasting model is shown in Figure 5 and comparison between actual value and forecasting value of passenger flow velocity based on spatioforecasting model is shown in Figure 6. 
TABLE 3: Average forecasting precision comparison of three approaches.

\begin{tabular}{|c|c|c|c|c|}
\hline Forecasting approach & $\begin{array}{l}\text { Average forecasting } \\
\text { precision of density }\end{array}$ & $\begin{array}{l}\text { Average CPU time of } \\
\text { density forecasting (s) }\end{array}$ & $\begin{array}{l}\text { Average forecasting } \\
\text { precision of velocity }\end{array}$ & $\begin{array}{l}\text { Average CPU time of } \\
\text { velocity forecasting (s) }\end{array}$ \\
\hline $\begin{array}{l}\text { Temporal forecasting approach based } \\
\text { on RBF NN }\end{array}$ & $95.21 \%$ & 9.8 & $94.73 \%$ & 9.6 \\
\hline $\begin{array}{l}\text { Spatio forecasting approach based on } \\
\text { spatial correlation degree }\end{array}$ & $91.23 \%$ & 11.2 & $88.21 \%$ & 12.1 \\
\hline $\begin{array}{l}\text { Hybrid temporal-spatio forecasting } \\
\text { approach }\end{array}$ & $96.83 \%$ & 12.9 & $96.10 \%$ & 13.7 \\
\hline
\end{tabular}

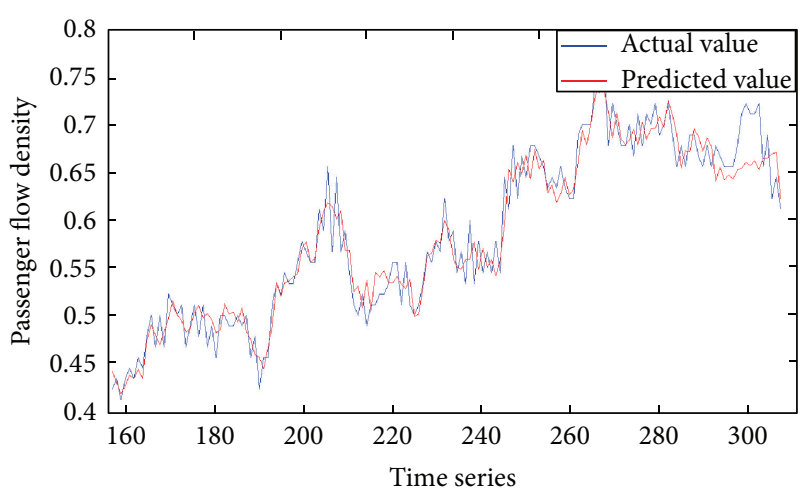

FIGURE 7: Comparion between actual value and forecasting value of passenger flow density based on hybrid temporal-spatio forecasting approach.

Based on the numerical fitting of actual and forecasting passenger flow status value in $P$, the forecasting value of passenger flow density based on hybrid temporal-spatio forecasting approach is calculated by (18) and the forecasting value of passenger flow velocity based on hybrid temporalspatio forecasting approach is calculated by (19). Consider

$$
\begin{aligned}
x_{\rho}(t)= & 0.889 x_{\rho r}(t)+\frac{0.034 \nu_{1}}{v_{0}} x_{\rho 1}(t-25) \\
& +\frac{0.050 v_{2}}{v_{0}} x_{\rho 2}(t-55) \\
& +\frac{0.0271 v_{3}}{v_{0}} x_{\rho 3}(t-20)+0.022, \\
x_{v}(t)= & 0.903 x_{v r}(t)+\frac{0.020 \rho_{1}}{\rho_{0}} x_{v 1}(t-35) \\
& +\frac{0.035 \rho_{2}}{\rho_{0}} x_{v 2}(t-55)+\frac{0.019 \rho_{3}}{\rho_{0}} x_{\nu 3}(t-20)+0.044 .
\end{aligned}
$$

The comparison between actual value and forecasting value of passenger flow density based on hybrid temporalspatio forecasting approach is shown in Figure 7 and comparison between actual value and forecasting value of passenger flow velocity based on hybrid temporal-spatio forecasting approach is shown in Figure 8.

The average forecasting precision comparison of three approaches mentioned above is shown in Table 3.

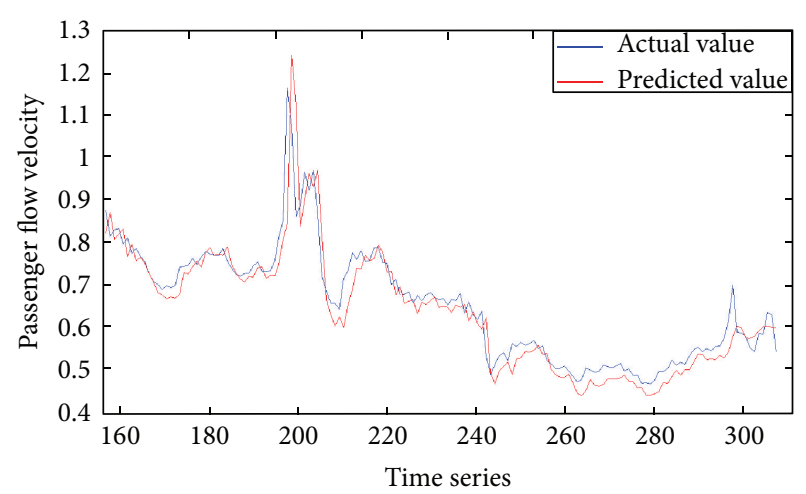

FIgURE 8: Comparion between actual value and forecasting value of passenger flow velocity based on hybrid temporal-spatio forecasting approach.

As observed in Table 3, the hybrid temporal-spatio forecasting approach proposed in this paper has the higher forecasting precisions than temporal and spatio forecasting approaches under the similar computation complexity. The results of computational experiments indicate that our approach is effective to forecast the passenger flow status of bottleneck positions in Chinese HRTH.

\section{Conclusion}

In this paper, we considered the forecasting approach for passenger flow status in the Chinese HRTH. A hybrid temporal-spatio forecasting approach was proposed, which combined temporal forecasting and spatio forecasting. The temporal forecasting based on RBF NN could fast and accurately forecast the status change of passenger flow but was insensitive for the influences from correlation points. A spatio forecasting approach based on spatial correlation degree was introduced to combine with the temporal forecasting approach to avoid the influences and improve the forecasting precision. Computational experiments on the actual passenger flow density and velocity from a specific bottleneck position and its correlation points in Chinese HRTH showed that the approach proposed in this paper is effective to forecast the passenger flow status of bottleneck position in HRTH with high forecasting precision for different types of passenger flow status. In the future, considering the passenger flow abnormal status forewarning of bottleneck position 
based on the passenger flow status forecasting is a possibility for further research.

\section{Conflict of Interests}

The authors declare that there is no conflict of interests regarding the publication of this paper.

\section{Acknowledgment}

This research was supported by a Grant (no. I11A300010) from the National Natural Science Foundation of China.

\section{References}

[1] W. Wang, W. Zhang, H. Guo, H. Bubb, and K. Ikeuchi, "A safetybased approaching behavioural model with various driving characteristics," Transportation Research C, vol. 19, no. 6, pp. 1202-1214, 2011.

[2] H. Guo, W. Wang, W. Guo, X. Jiang, and H. Bubb, "Reliability analysis of pedestrian safety crossing in urban traffic environment," Safety Science, vol. 50, no. 4, pp. 968-973, 2012.

[3] P. G. Gipps and B. Marksjö, "A micro-simulation model for pedestrian flows," Mathematics and Computers in Simulation, vol. 27, no. 2-3, pp. 95-105, 1985.

[4] A. Seyfried, B. Steffen, and T. Lippert, "Basics of modelling the pedestrian flow," Physica A, vol. 368, no. 1, pp. 232-238, 2006.

[5] H.-F. Jia, L.-L. Yang, and M. Tang, "Pedestrian flow characteristics analysis and model parameter calibration in comprehensive transport terminal," Journal of Transportation Systems Engineering and Information Technology, vol. 9, no. 5, pp. 117-123, 2009.

[6] X. Ji, X. Zhou, and B. Ran, "A cell-based study on pedestrian acceleration and overtaking in a transfer station corridor," Physica A, vol. 392, no. 8, pp. 1828-1839, 2013.

[7] L. Wang, Q. Zhang, Y. Cai, J. L. Zhang, and Q. G. Ma, "Simulation study of pedestrian flow in a station hall during the spring festival travel rush," Physica A, vol. 392, no. 10, pp. 24702478, 2013.

[8] M. Zhong, C. Shi, X. Tu, T. Fu, and L. He, "Study of the human evacuation simulation of metro fire safety analysis in China," Journal of Loss Prevention in the Process Industries, vol. 21, no. 3 , pp. 287-298, 2008.

[9] C. S. Jiang, F. Yuan, and W. K. Chow, "Effect of varying two key parameters in simulating evacuation for subway stations in China," Safety Science, vol. 48, no. 4, pp. 445-451, 2010.

[10] L. D. VanLandegen and X. Chen, "Microsimulation of largescale evacuations utilizing metrorail transit," Applied Geography, vol. 32, no. 2, pp. 787-797, 2012.

[11] C. Shi, M. Zhong, X. Nong, L. He, J. Shi, and G. Feng, "Modeling and safety strategy of passenger evacuation in a metro station in China," Safety Science, vol. 50, no. 5, pp. 1319-1332, 2012.

[12] J. Bi, S. Shao, W. Guan, and L. Wang, "State of charge estimation of Li-ion batteries in an electric vehicle based on a radial-basisfunction neural network," Chinese Physics B, vol. 21, no. 11, Article ID 118801, 2012.

[13] W. Y. Chang, "Equivalent circuit parameters estimation for PEM fuel cell using RBF neural network and enhanced particle swarm optimization," Mathematical Problems in Engineering, vol. 2013, Article ID 672681, 8 pages, 2013.
[14] E. E. Abusham and E. K. Wong, "Locally linear discriminate embedding for face recognition," Discrete Dynamics in Nature and Society, vol. 2009, Article ID 916382, 8 pages, 2009.

[15] J.-D. Wu and J.-C. Liu, "A forecasting system for car fuel consumption using a radial basis function neural network," Expert Systems with Applications, vol. 39, no. 2, pp. 1883-1888, 2012. 


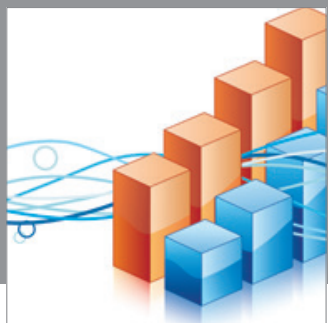

Advances in

Operations Research

mansans

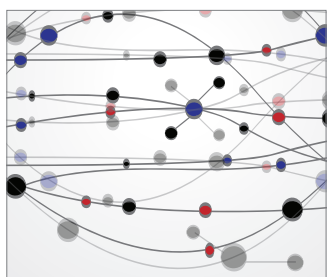

The Scientific World Journal
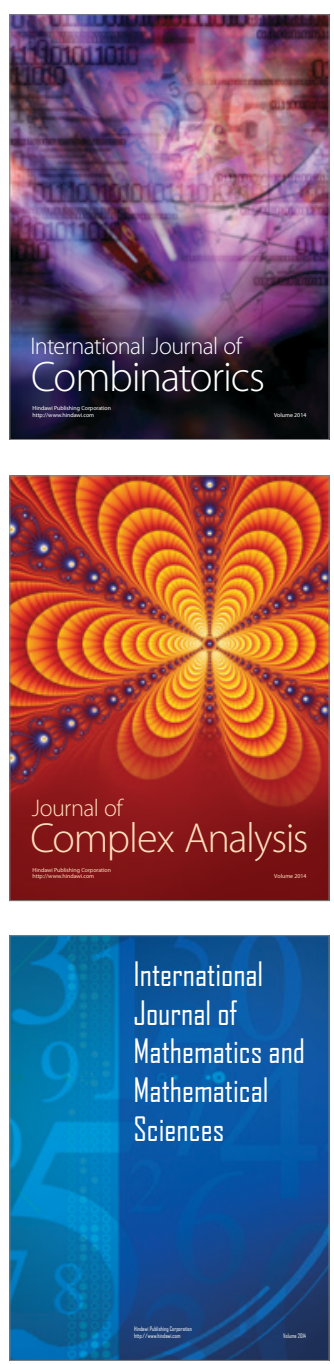
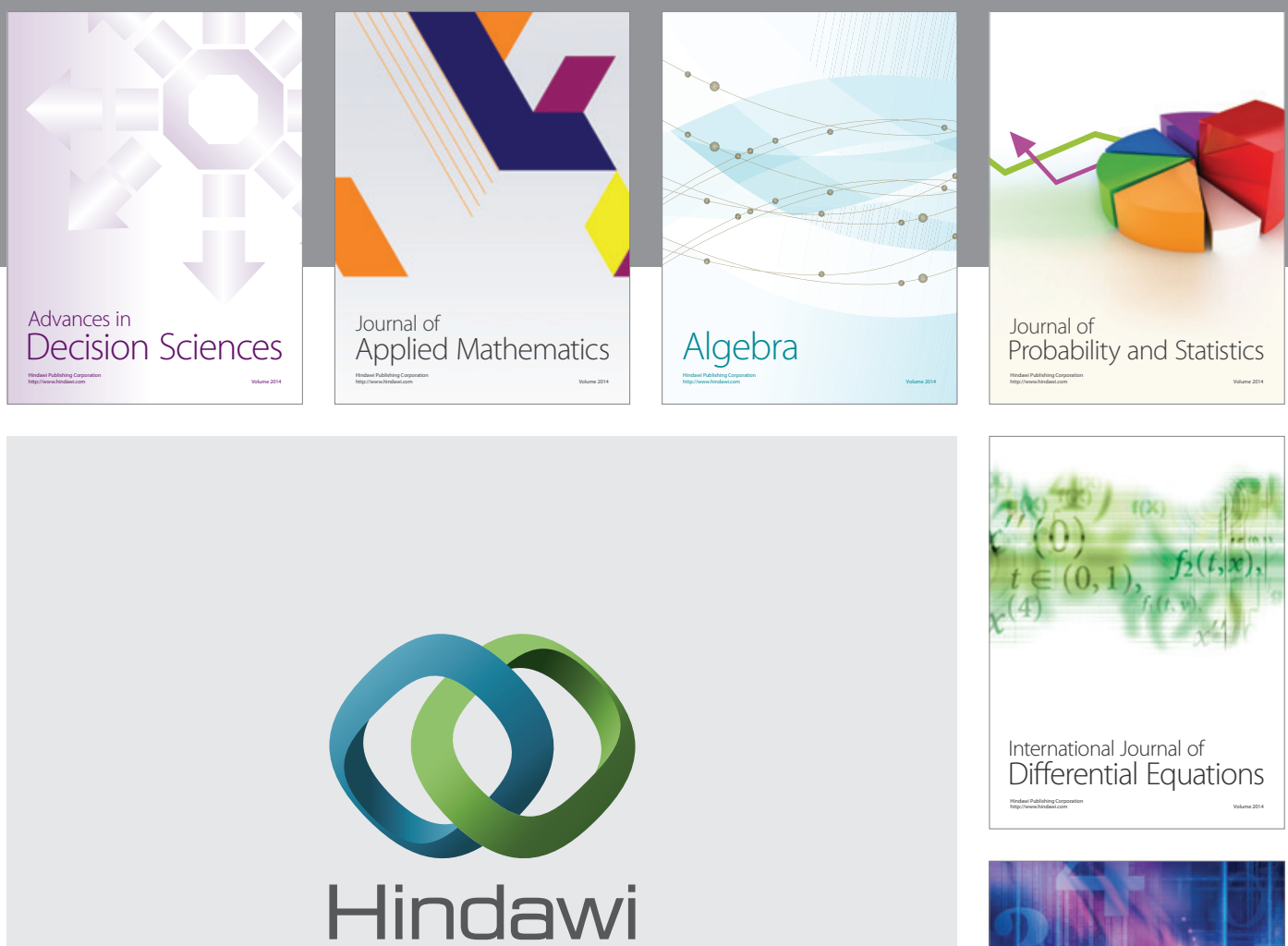

Submit your manuscripts at http://www.hindawi.com
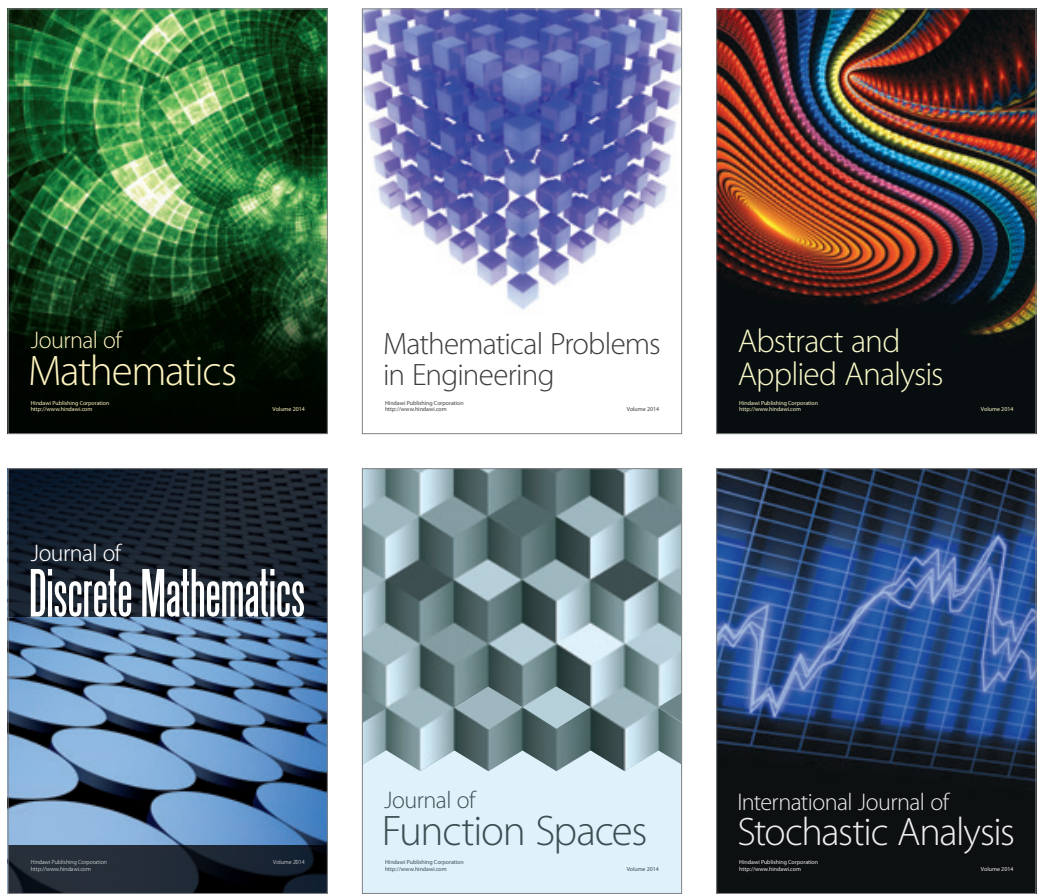

Journal of

Function Spaces

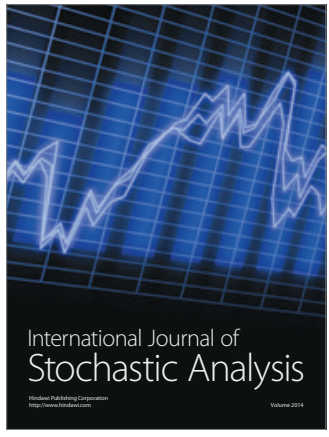

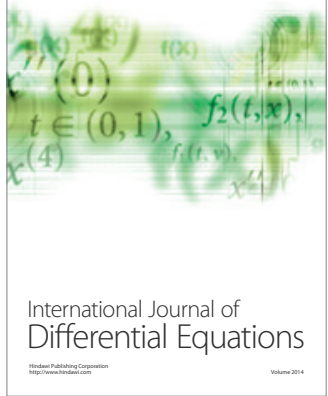
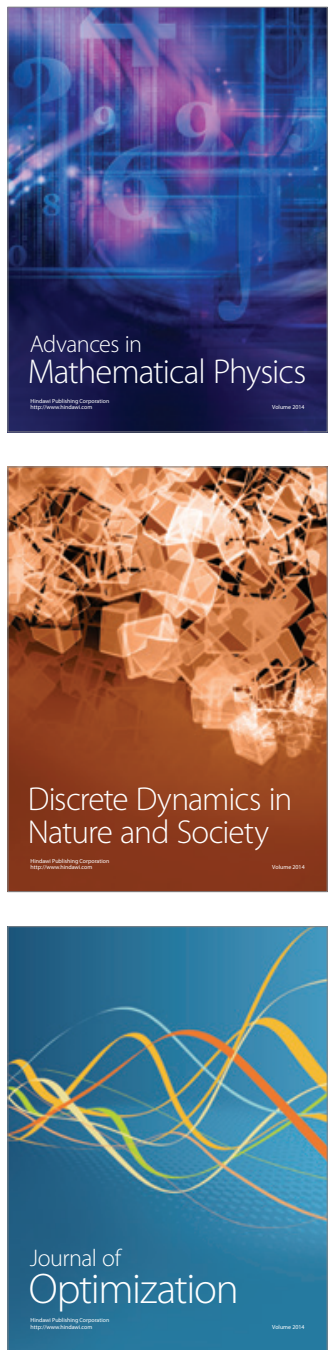